\title{
Visual Selective Attention in Adults with ADHD: Electrophysiological Evidence of Facilitation and Suppression
}

\author{
Jane W. Couperus*, Brittany R. Alperin, Dylan Furlong, Katherine Mott \\ Hampshire College, Amherst, USA \\ Email: “jcouperus@hampshire.edu
}

Received 10 February 2014; revised 12 March 2014; accepted 20 March 2014

Copyright $@ 2014$ by authors and Scientific Research Publishing Inc.

This work is licensed under the Creative Commons Attribution International License (CC BY).

http://creativecommons.org/licenses/by/4.0/

(c) (i) Open Access

\begin{abstract}
This study examined the functioning of facilitation and suppression in visual selective attention in adults with Attention Deficit Hyperactivity Disorder (ADHD). Electrophysiological data were examined in control adults and adults with ADHD during a cued visual spatial selective attention task where in addition to directing attention, the cue probabilistically predicted the presence of a distractor. Late activity following the cue (ADAN) showed attention modulation for all participants. However, only ADHD participants showed additional significant distractor anticipation modulations. Stimulus related activity showed modulations of early visual processing (P1) as a function of attention and anticipated distractor presence for all participants suggesting functional facilitation and suppression. Findings suggest functional visual spatial selective attention in adults with ADHD.
\end{abstract}

\section{Keywords}

Adult ADHD; Event Related Potentials; Visuospatial Selective Attention; P1; ADAN

\section{Introduction}

While often thought of as a disorder of childhood, attention deficit hyperactivity disorder (ADHD) is a significant challenge for adults as well. Adults with ADHD show higher levels of risk taking behavior [1], as well as higher rates of depression/anxiety, increased financial stress, and lower ratings on measures of relationship quality, health, and well-being [2]. However, many aspects of this disorder are still not well understood. For example, impairments in sustained attention and inhibition are thought to be primary deficits of the disorder [3]-[5].

\footnotetext{
"Corresponding author.
} 
However, a recent meta-analysis of studies using the continuous performance task (CPT), the most common test of sustained attention, by Huang-Pollock, Karalunas, Tam, and Moore [6] suggests that decrements in performance by those with ADHD are complex. For example, among other key factors that explain deficits in performance for those with ADHD are decreases in perceptual sensitivity (i.e. the ability to distinguish targets from distractors) and slower drift rates (i.e. the time it takes to gain the necessary information from encoding to make a decision). Moreover, other aspects of attention, such as selective attention, have been studied far less, and as a result present even greater challenges for developing a complete understanding of the disorder.

Endogenous (i.e. top-down) visual selective attention is a multi-faceted process, several aspects of which may be impacted by ADHD. Traditionally, endogenously driven attention has been described through a sensory gain model [7]. This theory suggests that signal enhancement works as a gain control, increasing the sensitivity of neurons to properties of the attended stimulus, relatively enhancing processing of stimuli at attended locations as compared to unattended locations [7]-[13]. Facilitation may work through control mechanisms active both prior to the onset of the stimulus, (i.e., preparatory facilitation seen in electrophysiological components such as the anterior directing attention negativity (ADAN)), as well as during stimulus processing (i.e., stimulus facilitation at electrophysiological components such as the P1; [14]). However, recent research suggests that suppressive mechanisms may also contribute to visual selective attention [15]. Suppression may contribute to selection by reducing the sensitivity of neurons to the properties or spatial location of the unattended stimulus both through control mechanisms prior to the onset of a distractor (preparatory suppression), as well as during stimulus processing (stimulus suppression) similar to facilitation. However, only facilitation processes have been examined in those with ADHD.

Much of the research on facilitation in selective attention in those with ADHD suggests that in many respects it functions similarly to typically developing populations [16]-[18]. When deficits in facilitation are seen, they occur primarily during stimulus processing (i.e. stimulus facilitation) at early levels of perceptual processing, typically examined in the P1 visual component [19]-[23].

In addition to potential differences seen in stimulus facilitation, there are remaining questions concerning possible processing differences in preparatory facilitation (e.g. activity following a spatial cue to the attended location in preparation for the stimulus). Few previous studies have examined preparatory evoked potentials related to facilitation that could account for differences in stimulus processing. When potential differences are examined, these studies have looked at the contingent negative variation (CNV) which is associated with anticipation or preparation and may reflect preparatory facilitation, but it is more typically associated with motor preparation. Moreover, the results from examining the CNV have been mixed, with some finding deficits [21] [24] [25] while others do not [26] [27]. In addition, none of these studies has examined frontal evoked potentials related specifically to attentional control seen in preparatory facilitation in visual spatial attention. Specifically, the attention directing anterior negativity (ADAN) is thought to reflect activity related to higher order attentional control for visual spatial attention [28] [29] and thus it is an ideal candidate for examining the functionality of preparatory facilitation in those with ADHD. However, to date, this component has not been examined in participants with ADHD.

In contrast to evidence of facilitation, evidence of suppression is more limited but growing [15] [30]-[34]. For example, a study by Couperus and Mangun [15] used a spatial cuing paradigm where target and distractor processing could be examined by utilizing unilateral (target only) and bilateral (target and distractor) displays. By cuing the location of the target stimulus as well as providing predictive information about the presence of distractors (validly cued $70 \%$ of the time, invalidly cued $30 \%$ of the time) the effects of distractor expectancy on attentional control as well as on target and distractor processing could be examined. Results suggest that changes in processing occur both prior to and at the onset of visual stimuli to both increase processing of attended stimuli (preparatory and stimulus facilitation) and reduce processing of unattended stimuli (preparatory and stimulus suppression, [15]).

This paradigm is particularly intriguing in the context of visual selective attention and its functioning in those with ADHD because it allows for a more detailed examination of the multiple aspects of visual selective attention that may be altered in those with ADHD. In particular, it allows for examination of three different aspects of visual selective attention (preparatory facilitation, preparatory suppression, and stimulus suppression) that have not been examined in this population. Understanding these different aspects of selective attention may help to explain how it is possible that those with ADHD are able to compensate behaviorally in visual selective attention tasks [3] as well as to help understand ADHD more completely. It may also provide additional evidence in 
support of this four-component model of selective attention (i.e. visual spatial selective attention being supported by four processes, preparatory facilitation, preparatory suppression, stimulus facilitation, and stimulus suppression).

Additionally, in contrast to children, there have been few studies of visual selective attention in adults with ADHD [22] [35] [36]. Moreover, the studies that do exist have not focused on the neurological correlates of visual selective attention but rather behavioral differences. Understanding the neurological processes underlying these differences in adults is critical for developing an understanding of ADHD more broadly as the rates of ADHD in adults have increased substantially over the last decade ${ }^{1}$ [37].

Therefore, this study will use the paradigm from Couperus and Mangun [15] to explore what mechanisms within visual selective attention (if any) may differ in those with ADHD, specifically in adults with ADHD. Two components that have previously shown facilitation and suppression effects, the ADAN and the P1 will be examined. The amplitude of the ADAN, as noted previously, is thought to correlate with activity in areas of the frontal cortex related to the control of visuospatial attention [29] and is typically seen in the contralateral hemisphere to the cued location following a directional cue (preparatory facilitation), but has also been shown to increase contralateral to an anticipated distractor (preparatory suppression, [15]). In contrast, the P1 is a component associated with visual processing in extrastriate cortex that is affected by visual attention (and may show both stimulus facilitation and suppression). Based on previous findings it is anticipated that both attention and the expectation of a distractor will alter activity at the ADAN for adult control participants reflecting preparatory facilitation and preparatory suppression [15]. Moreover, we anticipate that these participants will show both typical attentional stimulus facilitation at the P1 contralateral to target stimulus, and stimulus suppression contralateral to distractor stimulus when the cue indicates the presence of a distractor. For ADHD participants, we anticipate early reductions in processing of attended target stimuli at the P1 as compared to control participants (i.e. reduced stimulus facilitation, [21]). However, as no studies have examined the ADAN preparatory component nor suppressive activity at the $\mathrm{P} 1$, it is not clear if any differences in these components will be seen between groups.

\section{Methods}

\subsection{Participants}

Twenty-five adults diagnosed with attention deficit hyperactivity disorder (mean age $=20.44, \mathrm{SD}=2.28,7 \mathrm{Fe}-$ male) and 25 control participants (mean age $=21.00$, SD $=2.86,13$ Female) participated in this study from an initial 70 who were recruited (20 were excluded prior to and during data analysis based on exclusion criteria, 10 of which were control participants). Participants were recruited from the 5-College Consortium in the Pioneer Valley of Massachusetts (Amherst College, Smith College, Hampshire College, Mt. Holyoke College, and the University of Massachusetts at Amherst). All participants were right handed, were not born prematurely, and were not on psychotropic medications aside from stimulant medications (nine participants with ADHD were currently using stimulant medications regularly but none had taken any less than 18 hours before participation). All participants gave written consent prior to participation. All consents and research procedures were approved by the Hampshire College Institutional Review Board. Control participants were defined as those that met the above criteria as well as having indicated via self-report that they had never been diagnosed with or suspected of having a learning disability including specifically ADHD (and additionally not on stimulant or other psychotropic medications). Adults with ADHD who were currently taking stimulant medications (defined as having taken a stimulant medication within the last month, $n=9$ ) were asked to refrain from taking their medications at least 12 hours prior to participation in the study (no one reported use less than 18 hours prior to participation and there was an average time since last usage of 25.11 hours). To avoid and/or reduce altering participant's daily routine of medications most ADHD participants currently taking medication completed the study in the morning ${ }^{2}$. Assignment to the ADHD group was through both self-report (participants were only included if they indicated they had previously been diagnosed by a clinician with ADHD) and confirmed through the use of the Connor's Adult ADHD Ratings Scales. For inclusion adults had to present with significant symptoms (t-score above 65 on the total ADHD

\footnotetext{
${ }^{1}$ It should be noted that the reasons for the increase may be due to a number of factors including changing criteria, a stronger understanding of ADHD as a lifelong disorder, greater awareness of ADHD in adults, and many other reasons.

${ }^{2}$ There was no significant difference between groups in the time the study took place as a number of control participants also participated in the morning.
} 
symptoms subscale) on the Connor's Adult ADHD Ratings Scales ( $n=23$, mean $=78.74$, SD $=9.15)$. Connors ratings were not available for a subset of participants $(n=2)$ so to ensure data did not vary as a function of these participants all analyses were run both including and excluding these participants to ensure findings did not change.

\subsection{Selective Attention Task}

The selective attention task was based on the task used in Couperus and Mangun [15]. Participants were asked to complete 1000 trials (presented in blocks of 100). On each task trial, a cue (consisting of a set of two arrows, each arrow a different color, $0.5 \times 1.0$ degrees of visual angle) was presented for $150 \mathrm{~ms}$. The cue was followed by a random interstimulus interval of 1000 to $1300 \mathrm{~ms}$, and then the stimulus display was presented for $100 \mathrm{~ms}$ followed by a blank screen. The participant was encouraged to respond by pushing a response button rapidly and accurately. The next trial did not begin until after the participant had responded followed by an intertrial interval (response to next cue) that varied from 200 to 500 ms (see Figure 1).

There were two types of target stimulus displays in this paradigm; a unilateral (target only) and a bilateral (target plus distractor) display. The bilateral display consisted of two arrays of seven circular objects each (1.3 degrees for each circle). Each circle was half white and half black and was presented on a gray background that was isoluminant with the space-averaged luminance of the circles. The circle sets were 8 degrees from fixation measured to the center of the set, and each set subtended $4.6 \times 4.2$ degrees visual angle. The participant was asked to maintain fixation on a central fixation marker, covertly attend to one side of the display, and indicate if the center circle of the array was black in the upper half (right mouse button) or in the lower half of the circle (left mouse button) using a button press. The remaining 6 flanking circles consisted to two lateral flanking circles that were congruent in orientation to the central circle, two (one above, one below) that were in incongruent orientations, and two that were presented in a vertical orientation. The flanking circle orientations were chosen to create a complex stimulus to encourage selective attention while maintaining accuracy above 50\%. However, the orientations of these flanking stimuli remained consistent in relation to the central target stimulus across all trials as to avoid introducing additional influences on response choice. Circles presented on the unattended side appeared only in a vertical orientation, thus containing no information regarding the correct response. The unilateral display contained only circles on the attended (cued) side of the display. The cue presented prior to the onset of each display provided two pieces of information. It accurately informed the participant of the hemifield in which the target would appear (probability 1.0), and predicted whether a distractor was likely to appear in the display (probability 0.70 ). Thus, the location of the target, and therefore of covert attention, was correctly signaled by the cue, while the presence of the distractor was probablistically predicted by the cue. The cue arrow was always

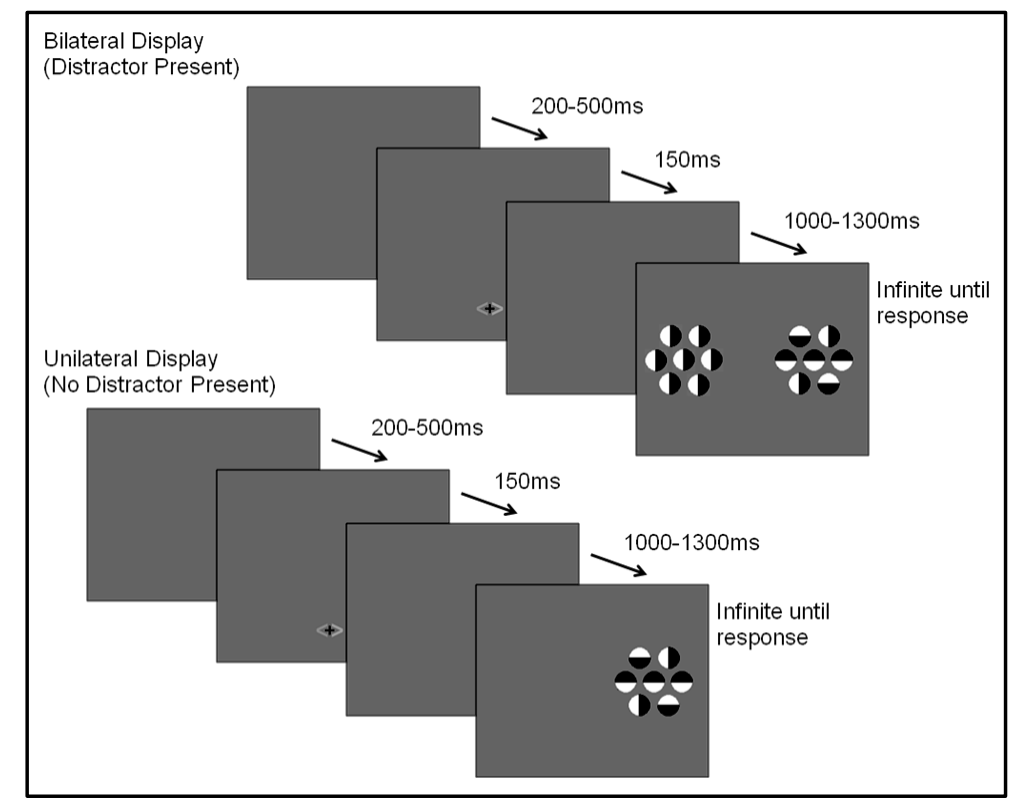

Figure 1. Task cue and stimulus displays. 
orange on one side and either blue or green on the other; the color blue or green indicated both the side of the target and if a distractor would be present (blue indicating a distractor would not be present, green indicating a distractor would be present).

\subsection{Electrophysiological Methods}

Scalp electroencephalograms (EEGs) were recorded using tin electrodes embedded in an elastic cap (Electro-cap International). The 32 electrodes were located at standard sites of the International 5 - 20 system of electrode placement [38] as follows: FZ, FPZ, CZ, CPZ, PZ, OZ, FP1, FP2, F7, F8, F3, F4, FT7, FT8, T7, T8, C3, C4, TP7, TP8, CP3, CP4, P7, P8, P3, P4, O1, O2, HEOG, VEOG. Electroencephalograms were recorded and referenced to the right mastoid and impedances were kept below 5k ohms for all participants. The mastoid reference is preferred when using a smaller number of channels because an average reference (mean of recorded electrodes) is not as accurate under such conditions [39]. The EEGs were amplified using a Synamps 2 Amplifier with a bandpass filter of 0.1 to $100 \mathrm{~Hz}$, and digitized at a sampling rate of 500 samples per second. To ensure eye fixation, electrooculograms (EOG) were recorded for both vertical and horizontal eye movements (electrodes were placed inferior to the left eye and both left and right of the outer canthus).

\subsection{Data Analysis and Reduction}

Behavioral data was averaged across attention location (left or right hemifield) to create four conditions based on cue validity and actual distractor presence: distractor cue valid/distractor present, distractor cue valid/distractor absent, distractor cue invalid/distractor present, and distractor cue invalid/distractor absent. Two participants were excluded from accuracy analysis due to reversing the meaning of the buttons for responding resulting in inaccurate accuracy. Reaction time and accuracy data were analyzed using repeated measures ANOVAs.

EEGs were re-referenced off-line to linked mastoids, sorted into epochs (200 ms pre-cue to $1000 \mathrm{~ms}$ post-cue and $200 \mathrm{~ms}$ pre-stimulus to $1000 \mathrm{~ms}$ post-stimulus), and artifact-free trials were averaged to yield ERPs for the different cue and stimulus types in the various conditions. The ERPs were baseline corrected using the mean of the 200 ms pre-stimulus period. Artifact rejection involved the automated exclusion of trials if they contained significant ocular artifacts (defined as amplitudes $+/-50 \mu$ volts at vertical or horizontal eye electrodes), muscle, or movement artifacts. Channels that were consistently bad across the experiment were marked as such and not used in analyses. Participants were eliminated from analyses if $10 \%$ or more channels were bad [40] or had less than 25 artifact free trials in any of the analyzed conditions (average number of trials included for cue displays = $118.85, \mathrm{SD}=35.70$, stimulus displays $=61.08, \mathrm{SD}=23.38$ ).

Cue-evoked waveforms were computed for each participant for the attention task for the two cue conditions directing attention to each side of the display: distractor present, distractor absent. The ADAN was chosen based on findings by Couperus and Mangun [15] that this component is sensitive to preparatory facilitation and preparatory suppression in this paradigm. Statistical analysis of preparatory activity as seen in the ADAN were based on mean amplitude measured over a 100-msec time window from 360 - $460 \mathrm{~ms}$, centered approximately on the peak amplitude of the component seen in the grand-averaged waveforms of the left and right frontal leads (F3/F4 and F7/F8). Additionally, we explored the possibility of effects on a second component sometimes associated with preparatory facilitation, the late directing positivity (LDAP), but no significant effects were seen (p’s $>0.05$ ) thus they are not reported here.

Stimulus-evoked waveforms were computed for the P1 for only bilateral displays for each of the two conditions directing attention to each side of the display: cue valid and cue invalid. Statistical analysis of stimulus related evoked potentials was based on mean amplitude measured over a 50-msec time window, centered approximately on the peak amplitude of the P1 (120 - 170) component seen in the grand-averaged waveforms of the left and right occipital leads (O1/O2). As with the ADAN, the P1 was chosen as it was sensitive to attention modulation in Couperus and Mangun [15] showing both stimulus facilitation and stimulus suppression. While there were also significant findings at the $\mathrm{N} 1$ (e.g. an interaction between cue validity (valid vs. invalid), cue direction (left vs. right), and electrode $(\mathrm{O} 1 \mathrm{vs.} \mathrm{O} 2)((F(1,44)=6.70, p=0.013)$, visualization of waveform subtractions suggest these findings reflect modulations at P1 that carry over to N1 rather than additional attention effects.

Repeated measures ANOVAs were used to examine the ADAN as well as P1 as a function of attention and distractor anticipation as well as comparing those with ADHD to control participants. 


\section{Results}

\subsection{Behavioral Analyses}

Reaction time and accuracy data were examined to determine if distractor anticipation influenced behavior as a function of cue validity and actual distractor presence. It is important to remember that distractor cue validity refers to the information in the cue regarding the presence/absence of distractors ( $70 \%$ valid, $30 \%$ invalid) as the cue was $100 \%$ predictive of the attended hemifield. A 2 (group: ADHD vs. control) by 2 (distractor cue validity: valid vs. invalid) by 2 (actual distractor presence: present vs. absent) repeated-measures ANOVA was used in analyses. Reaction time data showed a significant main effect of actual distractor presence $(F(1,48)=73.93, p<$ $0.001, \eta_{p}^{2}=0.606$; see Table 1$)$. The main effect demonstrates slower reaction times when a distractor was present. However, there were no differences as a function of group (ADHD versus control) and no significant interactions.

The results for accuracy (i.e. correctly indicating the color of the top half of the central circular stimulus of the attended array) showed the same effect, with greater accuracy for displays without a distractor compared to those with a distractor $\left(F(1,46)=11.94, p=0.001, \eta_{p}^{2}=0.206\right)$. As with reaction times there were no differences as a function of group and overall accuracy averaged 63.23 percent (SD $=13.2$ ) which is similar to previous studies using this paradigm [15]. There was, also a (nonsignificant) trend in the interaction between actual distractor presence and distractor cue validity $\left(F(1,46)=3.74, p=0.059, \eta_{p}^{2}=0.075\right)$. Although not significant, this interaction is consistent with findings in previous studies [30] which found higher accuracy when the presence of distractors was anticipated (a behavioral indicator of stimulus suppression). Thus, accuracy data suggest that subjects may utilize cue information concerning the likelihood that an upcoming display would contain a distractor.

\subsection{Late Preparatory Activity Following the Cue Reflected in the ADAN- 360 - $460 \mathrm{~ms}$ Post-Cue)}

To examine the ADAN, a 2 (group: ADHD vs. control) by 2 (cue distractor presence: present vs. absent) ${ }^{3}$ by 2 (cue direction: attend left vs. attend right) by 2 (electrode: F7 vs. F8 or F3 vs. F4) repeated-measures ANOVA was performed separately for the electrode pairs F3/F4 and F7/F8. While there were no main effects of group (ADHD versus control), cue distractor presence (i.e., present versus absent), or cue direction (indicating the hemifield of attention), there were several significant interactions. This analysis showed a significant effect of attention (shown through an interaction between cue direction and electrode with greater activity at the electrode contralateral to the cued direction; see Figure 2) for both electrode pairs (F3/F4, $F(1,48)=16.88, p<0.001$, $\left.\eta_{p}^{2}=0.260 ; \mathrm{F} 7 / \mathrm{F} 8, F(1,48)=6.29, p=0.016, \eta_{p}^{2}=0.116\right)$. Additionally, a significant interaction between the participant group (ADHD versus control), cue distractor presence and electrode was seen at the F3/F4 electrode pair $\left(\mathrm{F} 3 / \mathrm{F} 4, F(1,48)=4.58, p=0.037, \eta_{p}^{2}=0.087\right)$ as well as a three way interaction between cue distractor presence, cue direction, and electrode $\left(\mathrm{F} 3 / \mathrm{F} 4, F(1,48)=4.45, p=0.04, \eta_{p}^{2}=0.085\right)$. At F7/F8 there was only an additional main effect of electrode $\left(F(1,48)=7.61, p=0.008, \eta_{p}^{2}=0.137\right)$. To better understand the interactions at F3/F4, post-hoc analyses examined cue evoked potentials as a function of attention group. Contrary to previous findings [15] no main effect of cue distractor presence or interaction between cue distractor presence, cue direction, and electrode was found for control participants (p's $>0.05$ ) despite a clear effect of attention $\left(F(1,24)=12.30, p=0.002, \quad \eta_{p}^{2}=0.339\right.$, i.e. preparatory facilitation seen in Figure 2$)$ and presence in the raw waveforms of an effect of cue distractor presence (i.e. see increased ADAN to distractor present cues as compared to distractor absent cues in Figure 3). In contrast, the ADHD participants showed both this significant attention effect $\left(F(1,24)=4.80, p=0.038, \eta_{p}^{2}=0.167\right)$ reflecting preparatory facilitation and a significant 3 way interaction between cue distractor presence, cue direction, and electrode $(F(1,24)=5.90, p=0.023$, $\eta_{p}^{2}=0.197$ ) reflecting both preparatory facilitation and preparatory suppression. These effects appear to reflect greater activity at F4 when there was a cue directing attention to the left that also indicated a distractor would be present, although follow-up t-tests did not reveal any more specific significant differences. This increased positivity results in a negative peak (the ADAN) when ERPs produced by cues directed to the right are subtracted from those produced by cues directed to the left at F4.

\footnotetext{
${ }^{3}$ The term cue distractor presence is used here rather than distractor cue validity as the validity of the cue is not yet relevant as the participant does not become aware of the validity of the cue until the stimulus is present.
} 
Table 1. Reaction time and accuracy data.

\begin{tabular}{|c|c|c|c|c|}
\hline \multicolumn{5}{|c|}{ Reaction Time } \\
\hline & Cue Valid Distractor Present & Cue Valid Distractor Absent & Cue Invalid Distractor Present & Cue Invalid Distractor Absent \\
\hline Control & $729.61(128.0)$ & $637.51(97.4)$ & $727.61(115.7)$ & $674.05(96.4)$ \\
\hline ADHD & $780.36(161.3)$ & $715.06(146.4)$ & $783.82(172.0)$ & $724.52(147.3)$ \\
\hline \multicolumn{5}{|c|}{ Accuracy } \\
\hline & Cue Valid Distractor Present & Cue Valid Distractor Absent & Cue Invalid Distractor Present & Cue Invalid Distractor Absent \\
\hline Control & $63.2(12.2)$ & $63.9(12.8)$ & $63.0(12.6)$ & $64.7(14.4)$ \\
\hline ADHD & $62.2(14.3)$ & 63.3(15.3) & $61.0(13.5)$ & $64.6(14.1)$ \\
\hline
\end{tabular}

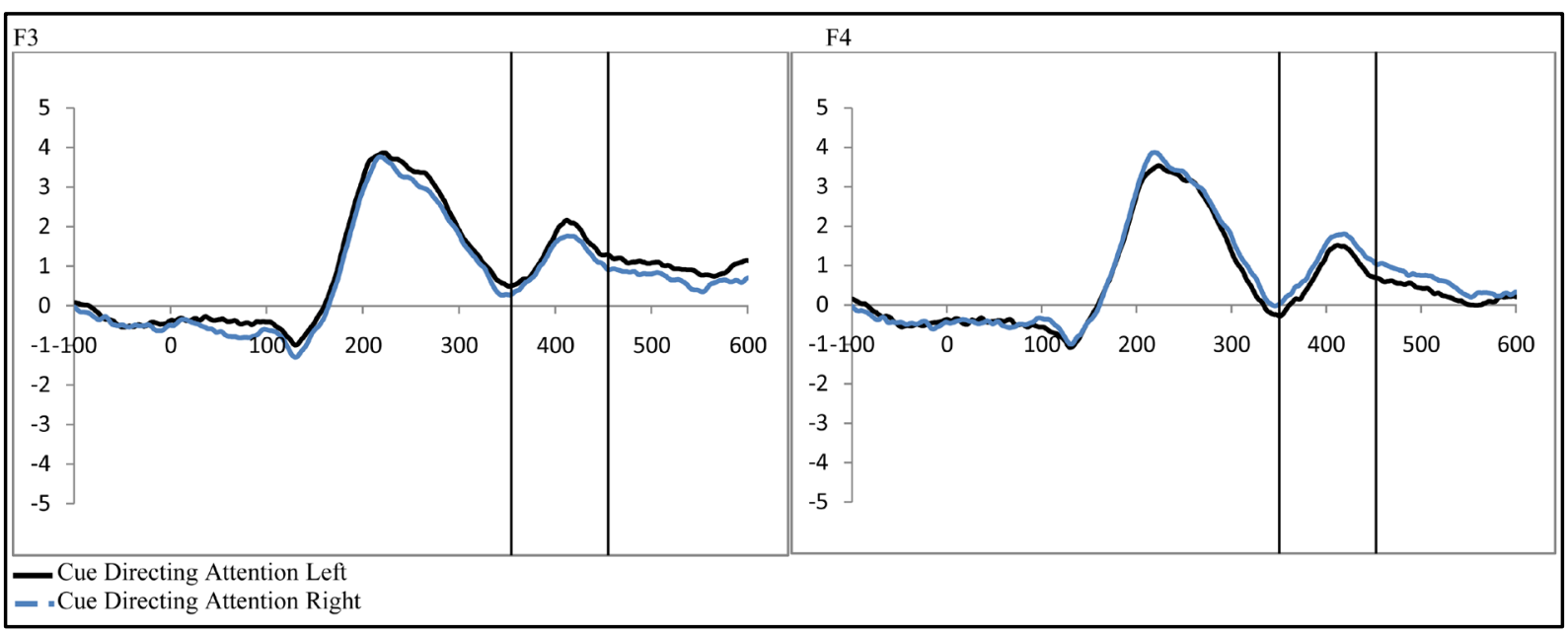

Figure 2. Late activity following the cue-ADAN (360 - $460 \mathrm{~ms}$ ). Collapsed across attention groups (ADHD and control), activity at frontal electrodes in response to the directional distractor cue to the left or right.

\subsection{Stimulus Processing at P1-(120 - 170 ms Post-Stimulus)}

To examine activity related to the bilateral stimulus display (containing both the attended target and unattended distractor), a 2 (group: ADHD vs. control) by 2 (distractor cue validity: valid vs. invalid) by 2 (cue direction: attend left vs. attend right) by 2 (electrode: $\mathrm{O} 1$ or O2) repeated-measures ANOVA was performed. While there were no main effects, there was a significant interaction between distractor cue validity, cue direction, and electrode $\left(\mathrm{O} 1 / \mathrm{O} 2, \mathrm{~F}(1,48)=23.14, p<0.001, \eta_{p}^{2}=0.325\right)$. Follow-up analysis suggests that this interaction stemsfrom attention effects at $\mathrm{O} 1$ that appear when a distractor is anticipated (distractor cue valid) as compared to when one is not anticipated (distractor cue invalid; O1cue valid, $t(49)=-2.99, p=0.004$, O1 distractor cue invalid $p>0.05$, O2's $p>0.05$; see Figure 4). Furthermore, the lack of attention effect when the distractor was not anticipated (distractor cue invalid) can be understood through the combined effects of facilitation and suppression. Specifically, there appears to be stimulus suppression of the unattended distractor at O1, with greater activity contralateral to the appearance of a distractor when that distractor was not anticipated compared to when it was anticipated (distractor cue valid vs. invalid; O1, $t(49)=-2.08, p=0.043$, see Figure 5). Moreover, stimulus facilitation of the attended stimulus is stronger when a distractor was anticipated as can be seen at $\mathrm{O} 1$ through greater processing contralateral to the target when a distractor was anticipated compared to when it was not (distractor cue valid vs. invalid; O1, $t(49)=2.75, p=0.008$ ). These combined effects modulate attention effects such that attention effects are primarily seen when a distractor is anticipated.

\section{Discussion}

Previous research suggests that any visual selective attention deficits in attention deficit hyperactivity disorder 


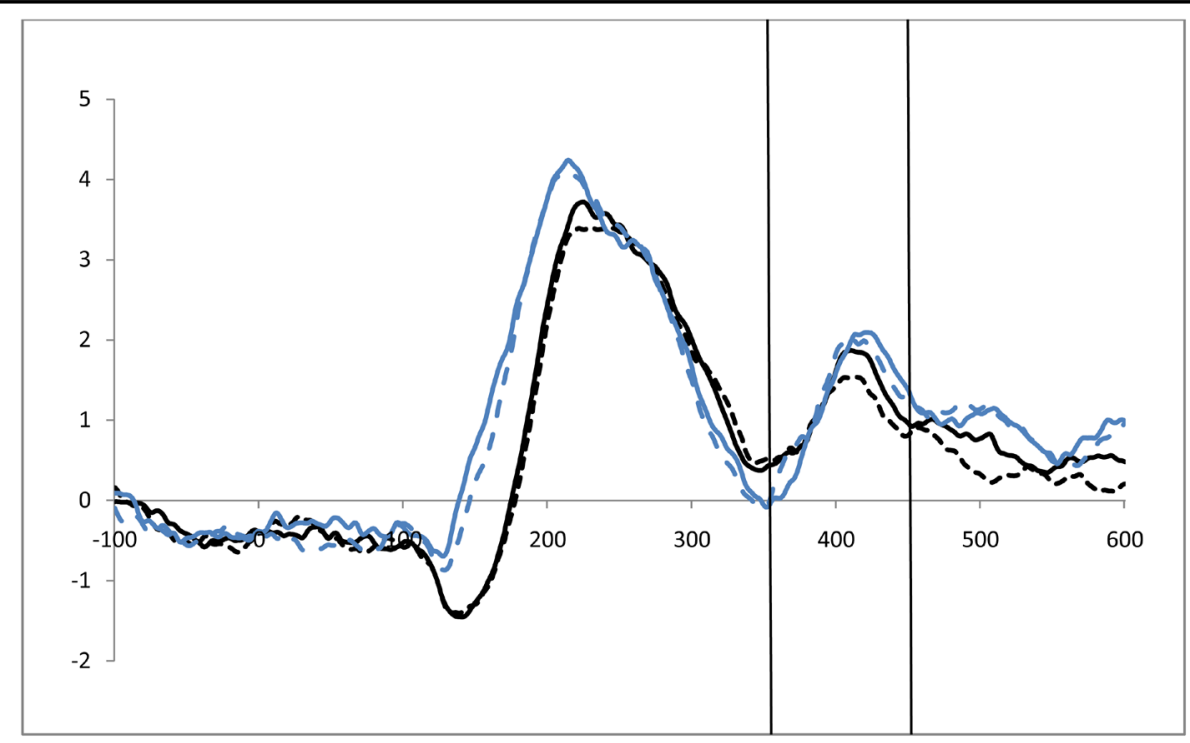

Control Cue Distractor Present

- Control Cue Distractor Absent

- ADHD Cue Distractor Present

- ADHD Cue Distractor Absent

Figure 3. Late activity following the cue-ADAN (360 - $460 \mathrm{~ms}$ ). Collapsed across cue direction (left vs. right) and electrode (F3/F4). It is important to note, that for the ADAN, attention effects are typically seen through a subtraction that results in a negativity when the activity contralateral to the unattended hemifield is subtracted from activity contralateral to the attended hemifield (Seiss et al., 2009), however raw waveforms are presented here to visualize differences between groups as a function of the cue to distractor presence.

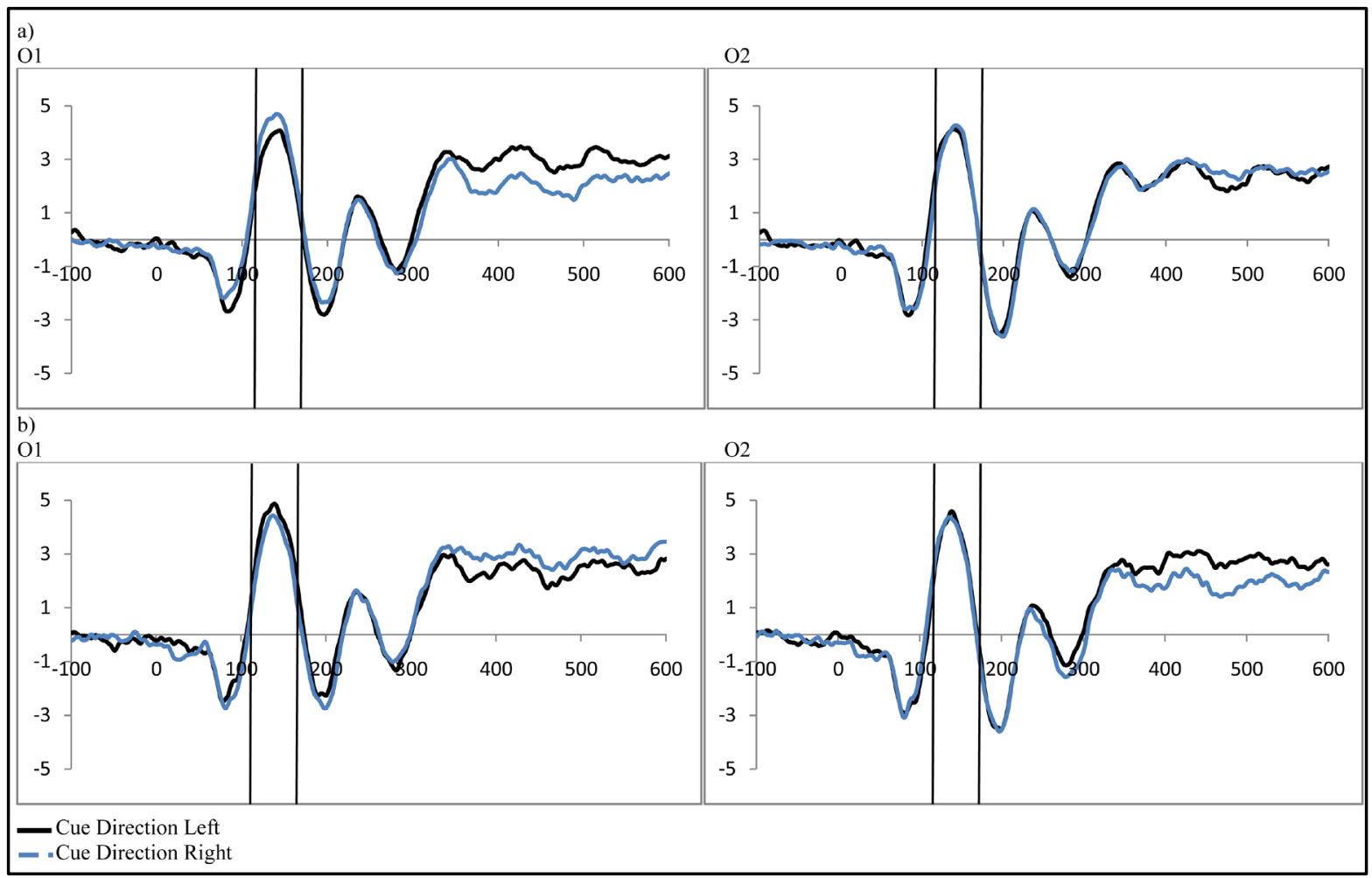

Figure 4. Activity in response to the stimulus at P1 as a function of attention when (a) a distractor was anticipated (distractor cue valid) and (b) when a distractor was not anticipated (distractor cue invalid; collapsed across group, control vs. ADHD). 


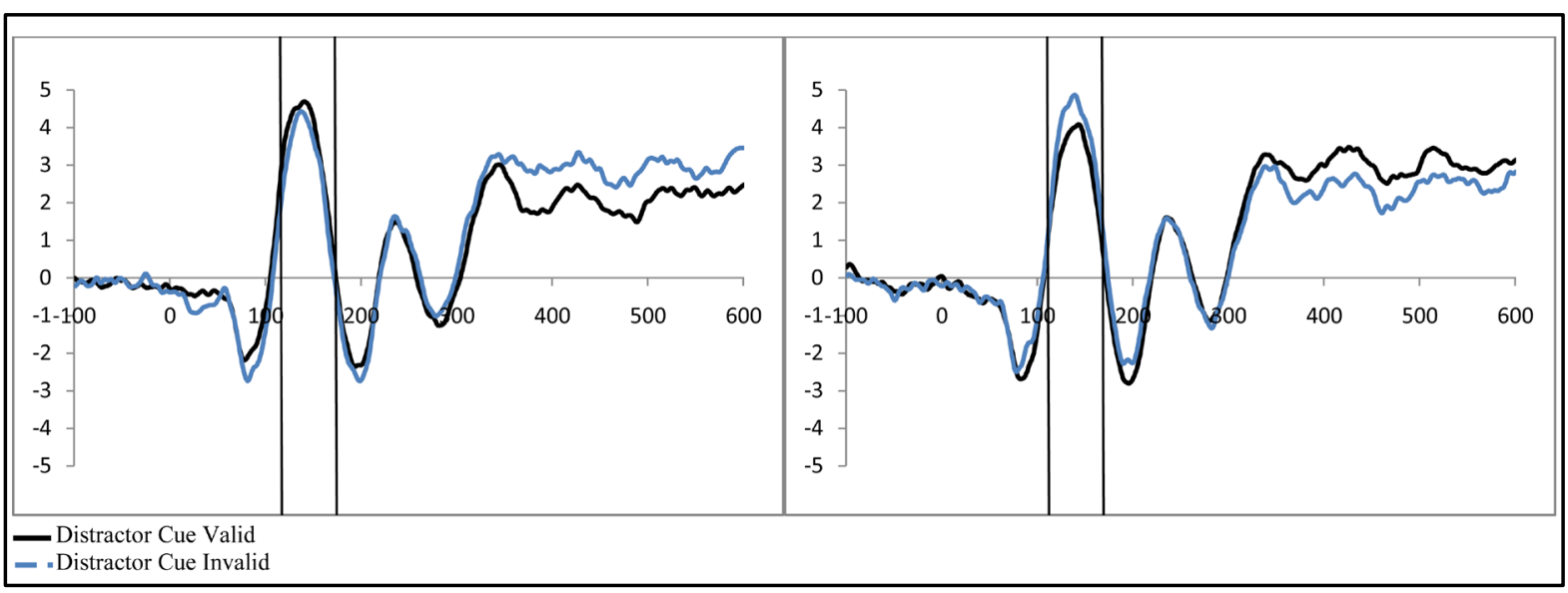

Figure 5. Example of P1 related activity as a function of distractor expectancy as seen at O1 (collapsed across group, control vs. ADHD). Greater activity is seen when (a) the distractor is validly cued contralateral to the attended stimulus (stimulus facilitation) and (b) reduced for the unattended stimulus when the distractor is validly cued (stimulus suppression).

are in early processing of stimuli [21]. However, the current study suggests that, while deficits in selective attention may exist in younger children, they do not appear to last into adulthood. Participants with ADHD in this study showed evidence of preparatory facilitation, preparatory suppression, stimulus facilitation, and stimulus suppression. Additionally, while findings suggest there may be some small differences in cue related evoked potentials (i.e. preparatory activity), no deficits appear in either preparatory or stimulus processing for adults. Thus findings suggest that deficits in visual spatial selective attention are either small and unstable, or as noted previously, they do not persist into adulthood.

Examination of late post cue evoked potentials suggests attention effects (i.e. increased activity contralateral to the cued hemifield) at the ADAN for both ADHD and control participants that reflect preparatory facilitation. This is consistent with previous studies with neurotypical participants [15]. Moreover, contrary to previous studies of other cue related activity such as the CNV [24], this is the first time such cue related attention effects have been shown in adults with ADHD.

However, the picture becomes a bit more complex when adding the role of distractor anticipation to general attention effects as it allows for an additional look at facilitation and also allows for the examination of suppression. Intriguingly, there were some interactions between activity associated with cues indicating a distractor would be present as compared to when no such distractor would be present that highlight the need for additional study of preparatory suppression. In contrast to previous findings [15], this study did not show effects of distractor anticipation on the ADAN for control participants that might indicate preparatory suppression and additional preparatory facilitation. However, as can be seen in Figure 3, while effects were not significant (possibly due to variability within the data), data were not opposed to previous findings suggesting that either this effect is small or it is unstable, indicating a need for further study. In contrast, ADHD participants did show an effect of distractor anticipation on attention modulations suggesting preparatory facilitation and suppression, although follow-up analysis did not find more specific significant effects. Detailed examination of the data suggests this effect may be a result of greater activity contralateral to the attended hemifield when a distractor was cued (i.e. preparatory facilitation). As previous studies with neurotypical adults primarily showed distractor anticipation effects contralateral to the unattended hemifield (i.e. preparatory suppression) it is possible there are differences in processing of the ADAN between those with ADHD and neurotypical adults. However, as no specific effects were significant in this study, only further study may determine if such differences exist.

Turning to stimulus processing, previous research suggests possible deficits in early perceptual processing in those with ADHD [21]. However, contrary to findings with children, this study did not show such deficits. Both control and ADHD participants demonstrated a significant attention effect at the P1 visual component. Additionally, the size of this effect did not differ as a function of attention group (control versus ADHD). Furthermore, a more detailed analysis of the effects of distractor anticipation shows that both control and ADHD participants engage in both stimulus facilitation and stimulus suppression. This was demonstrated through an increase in activity contralateral to the attended location as compared to activity contralateral to the unattended 
location (i.e. stimulus facilitation). Moreover, at $\mathrm{O} 1$ this increase in processing contralateral to the attended stimulus location was primarily seen when a distractor was anticipated as compared to when it was not. In contrast, processing contralateral to the unattended stimulus location was decreased (i.e. stimulus suppression) when a distractor was anticipated as compared to when it was not.

\section{Conclusion}

Findings from this study are important as they suggest that while some deficits in early visual selective attention may be seen in childhood, such deficits may not persist into adulthood. This is consistent with theories that suggest that ADHD may involve a developmental lag for some aspects of brain development. For example, Shaw et al. [41] found delays in the maturation of the cortex (as measured by cortical thickness) for those with ADHD compared to neurotypical controls across a wide age range $(5-20+)$. Thus, differences in findings between children and adults may reflect delayed development of areas supporting visual selective attention in those with ADHD as compared to control participants. Some studies [42] have begun to examine such changes over time and do suggest that different aspects of attention may show a developmental lag while others do not. However, these studies have not examined preparatory activity related to frontal attentional control or attention effects in early stimulus processing, such as those examined in this study. Thus, while findings of this study are evidence of possible lags in maturation, future studies should examine visual selective attention longitudinally and ideally correlate changes in attentional processes with maturation of the brain. Finally, given the relative paucity of research concerning the functioning of visual selective attention in those with ADHD, and even more limited studies done on adults with ADHD, this research helps to build a more complete understanding of the disorder.

\section{References}

[1] Matthies, S., Philipsen, A. and Svaldi, J. (2012) Risky Decision Making in Adults with ADHD. Journal of Behavior Therapy and Experimental Psychiatry, 43, 938-946. http://dx.doi.org/10.1016/j.jbtep.2012.02.002

[2] Das, D., Cherbuin, N., Butterworth, P., Anstey, K.J. and Easteal, S. (2012) A Population-Based Study of Attention Deficit/Hyperactivity Disorder Symptoms and Associated Impairment in Middle-Aged Adults. PloS One, 7, e31500. http://dx.doi.org/10.1371/journal.pone.0031500

[3] DeShazo Barry, T., Klinger, L.G., Lyman, R.D., Bush, D. and Hawkins, L. (2001) Visual Selective Attention versus Sustained Attention in Boys with Attention-Deficit/ Hyperactivity Disorder. Journal of Attention Disorders, 4, 193-202. http://dx.doi.org/10.1177/108705470100400401

[4] Booth, J.R., Burman, D.D., Meyer, J.R., Lei, Z., Trommer, B.L., Davenport, N.D. and Mesulam, M.M. (2005) Larger Deficits in Brain Networks for Response Inhibition than for Visual Selective Attention in Attention Deficit Hyperactivity Disorder (ADHD). Journal of Child Psychology and Psychiatry, and Allied Disciplines, 46, 94-111. http://dx.doi.org/10.1111/j.1469-7610.2004.00337.x

[5] Kiliç, B.G., Sener, S., Koçkar, A.I. and Karakaş, S. (2007) Multicomponent Attention Deficits in Attention Deficit Hyperactivity Disorder. Psychiatry and Clinical Neurosciences, 61, 142-148. http://dx.doi.org/10.1111/j.1440-1819.2007.01629.x

[6] Huang-Pollock, C.L., Karalunas, S.L., Tam, H. and Moore, A.N. (2012) Evaluating Vigilance Deficits in ADHD: A Meta-Analysis of CPT Performance. Journal of Abnormal Psychology, 121, 360-371. http://dx.doi.org/10.1037/a0027205

[7] Hillyard, S., Vogel, E. and Luck, S. (1998) Sensory Gain Control (Amplification) as a Mechanism of Selective Attention: Electrophysiological and Neuroimaging Evidence. Philosophical Transactions of the Royal Society of London. Series B: Biological Sciences, 353, 1257-1270. http://rstb.royalsocietypublishing.org/content/353/1373/1257.short http://dx.doi.org/10.1098/rstb.1998.0281

[8] Russo, F.Di, Martínez, A., Hillyard, S.A., Jolla, L., Lucia, F.S. and Universitario, I. (2003) Source Analysis of EventRelated Cortical Activity during Visuo-Spatial Attention. Cerebral Cortex, 486-499. http://dx.doi.org/10.1093/cercor/13.5.486

[9] Handy, T.C. and Khoe, W. (2005) Attention and Sensory Gain Control : A Peripheral Visual Process ? Journal of Cognitive Neuroscience, 2002, 1936-1949.

[10] Mangun, G.R. and Hillyard, S.A. (1991) Modulations of Sensory-Evoked Brain Potentials Indicate Changes in Perceptual Processing during Visual-Spatial Priming. Perception, 17, 1057-1074.

[11] Mangun, G. (1995) Neural Mechanisms of Visual Selective Attention. Psychophysiology. http://dx.doi.org/10.1111/j.1469-8986.1995.tb03400.x 
[12] Navalpakkam, V. and Itti, L. (2007) Article Search Goal Tunes Visual Features Optimally. Neuron, 53, 605-617. http://dx.doi.org/10.1016/j.neuron.2007.01.018

[13] Mangun, G.R. and Fannon, S.P. (2007) Attention: Control in the Visual Cortex. Current Biology, 17, 170-172. http://dx.doi.org/10.1016/j.cub.2007.01.002

[14] Dale, C.L., Simpson, G.V, Foxe, J.J., Luks, T.L. and Worden, M.S. (2008) ERP Correlates of Anticipatory Attention: Spatial and Non-Spatial Specificity and Relation to Subsequent Selective Attention. Experimental Brain Research, 188, 45-62. http://dx.doi.org/10.1007/s00221-008-1338-4

[15] Couperus, J.W. and Mangun, G.R. (2010) Signal Enhancement and Suppression during Visual-Spatial Selective Attention. Brain research, 1359, 155-177. http://dx.doi.org/10.1016/j.brainres.2010.08.076

[16] Huang-Pollock, C.L., Nigg, J.T. and Carr, T.H. (2005) Deficient Attention Is Hard to Find: Applying the Perceptual Load Model of Selective Attention to Attention Deficit Hyperactivity Disorder Subtypes. Journal of Child Psychology and Psychiatry, and Allied Disciplines, 46, 1211-1218. http://dx.doi.org/10.1111/j.1469-7610.2005.00410.x

[17] Douglas, V. (1999) Cognitive Control Processes in Attention-Deficit/Hyperactivity Disorder. In: Quay, H. and Hogan, A., Eds., Handbook of Disruptive Behavior Disorders, Kluwer Academic, New York, pp. 105-138. http://dx.doi.org/10.1007/978-1-4615-4881-2_5

[18] Sergeant, J., Oosterlaan, J. and van der Meere, J. (1999) NoInformation Processing and Energetic Factors in AttentionDeficit/Hyperactivity Disorder. In: Quay, H. and Hogan, A., Eds., Handbook of Disruptive Behavior Disorders, Kluwer Academic, New York, pp. 75-103. http://dx.doi.org/10.1007/978-1-4615-4881-2_4

[19] Gomes, H., Duff, M., Ramos, M., Molholm, S., Foxe, J.J. and Halperin, J. (2012) Auditory Selective Attention and Processing in Children with Attention-Deficit/Hyperactivity Disorder. Clinical Neurophysiology: Official Journal of the International Federation of Clinical Neurophysiology, 123, 293-302. http://dx.doi.org/10.1016/j.clinph.2011.07.030

[20] Jonkman, L.M., Kenemans, J.L., Kemner, C., Verbaten, M.N. and van Engeland, H. (2004) Dipole Source Localization of Event-Related Brain Activity Indicative of an Early Visual Selective Attention Deficit in ADHD Children. Clinical Neurophysiology: Official Journal of the International Federation of Clinical Neurophysiology, 115, 1537-1549. http://dx.doi.org/10.1016/j.clinph.2004.01.022

[21] Perchet, C., Revol, O., Fourneret, P., Mauguière, F. and Garcia-Larrea, L. (2001) Attention Shifts and Anticipatory Mechanisms in Hyperactive Children: An ERP Study Using the Posner Paradigm. Biological Psychiatry, 50, 44-57. http://www.ncbi.nlm.nih.gov/pubmed/11457423 http://dx.doi.org/10.1016/S0006-3223(00)01119-7

[22] Stevens, A.A., Maron, L., Nigg, J.T., Cheung, D., Ester, E.F. and Awh, E. (2012) Increased Sensitivity to Perceptual Interference in Adults with Attention Deficit Hyperactivity Disorder. Journal of the International Neuropsychological Society, 18, 511-520. http://dx.doi.org/10.1017/S1355617712000033

[23] Stelt, O., Van Der, Molen, M., Van Der, Gunning, W.B. and Kok, A. (2001) Neuroelectrical Signs of Selective Attention to Color in Boys with Attention-Deficit Hyperactivity Disorder. Preliminary Results of this Study Were Presented at the 39th Meeting of the Society for Psychophysiological Research (SPR), Granada, 6-10 October 1999, 12, 245-264.

[24] Banaschewski, T., Yordanova, J., Kolev, V., Heinrich, H., Albrecht, B. and Rothenberger, A. (2008) Stimulus Context and Motor Preparation in Attention-Deficit/Hyperactivity Disorder. Biological Psychology, 77, 53-62. http://dx.doi.org/10.1016/j.biopsycho.2007.09.003

[25] Sartory, G., Heine, A., Müller, B.W. and Elvermann-Hallner, A. (2002) Event- and Motor-Related Potentials during the Continuous Performance Task in Attention-Deficit/Hyperactivity Disorder. Journal of Psychophysiology, 16, 97-106. http://dx.doi.org/10.1027//0269-8803.16.2.97

[26] Strandburg, R.J., Marsh, J.T., Brown, W.S., Asarnow, R.F., Higa, J., Harper, R. and Guthrie, D. (1996) ContinuousProcessing-Related Event-Related Potentials in Children with Attention Deficit Hyperactivity Disorder. Biological Psychiatry, 40, 964-980. http://dx.doi.org/10.1016/0006-3223(95)00545-5

[27] Van Leeuwen, T.H., Steinhausen, H.C., Overtoom, C.C., Pascual-Marqui, R.D., van't Klooster, B., Rothenberger, A. and Brandeis, D. (1998) The Continuous Performance Test Revisited with Neuroelectric Mapping: Impaired Orienting in Children with Attention Deficits. Behavioural Brain Research, 94, 97-110. http://www.ncbi.nlm.nih.gov/pubmed/9708843 http://dx.doi.org/10.1016/S0166-4328(97)00173-3

[28] Nobre, A.C., Sebestyen, G.N. and Miniussi, C. (2000) The Dynamics of Shifting Visuospatial Attention Revealed by Event- Related Potentials. Neuropsychologia, 38, 964-974. http://dx.doi.org/10.1016/S0028-3932(00)00015-4

[29] Seiss, E., Gherri, E., Eardley, A.F. and Eimer, M. (2007) Do ERP Components Triggered during Attentional Orienting Represent Supramodal Attentional Control? Society, 44, 987-990. http://dx.doi.org/10.1111/j.1469-8986.2007.00591.x

[30] Awh, E., Matsukura, M. and Serences, J.T. (2003) Top-Down Control over Biased Competition during Covert Spatial Orienting. Journal of Experimental Psychology: Human Perception and Performance, 29, 52-63. http://dx.doi.org/10.1037/0096-1523.29.1.52 
[31] Braithwaite, J.J., Humphreys, G.W. and Hulleman, J. (2005) Color-Based Grouping and Inhibition in Visual Search: Evidence from a Probe Detection Analysis of Preview Search. Perception \& Psychophysics, 67, 81-101.

[32] Caputo, G. and Guerra, S. (1998) Attentional Selection by Distractor Suppression. Science, 38, 669-689.

[33] Hay, J.L., Milders, M.M. and Niedeggen, M. (2006) The Effect of Perceptual Load on Attention-Induced Motion Blindness: The Efficiency of Selective Inhibition. Journal of Experimental Psychology, 32, 885-907. http://dx.doi.org/10.1037/0096-1523.32.4.885

[34] Seiss, E., Driver, J. and Eimer, M. (2009) Clinical Neurophysiology Effects of Attentional Filtering Demands on Preparatory ERPs Elicited in a Spatial Cueing Task. Clinical Neurophysiology, 120, 1087-1095. http://dx.doi.org/10.1016/j.clinph.2009.03.016

[35] Biederman, J., Petty, C.R., Fried, R., Doyle, A.E., Spencer, T., Seidman, L.J. and Faraone, S.V. (2007) Stability of Executive Function Deficits into Young Adult Years: A Prospective Longitudinal Follow-Up Study of Grown up Males with ADHD. Acta Psychiatrica Scandinavica, 116, 129-136. http://dx.doi.org/10.1111/j.1600-0447.2007.01008.x

[36] Conzelmann, A., Pauli, P., Mucha, R.F., Jacob, C.P., Gerdes, A.B.M., Romanos, J. and Weyers, P. (2010) Early Attentional Deficits in an Attention-to-Prepulse Paradigm in ADHD Adults. Journal of Abnormal Psychology, 119, $594-603$. http://dx.doi.org/10.1037/a0019859

[37] Montejano, L., Sasané, R., Hodgkins, P., Russo, L. and Huse, D. (2011) Adult ADHD: Prevalence of Diagnosis in a US Population with Employer Health Insurance. Current Medical Research and Opinion, 27, 5-11. http://dx.doi.org/10.1185/03007995.2011.603302

[38] Jurcak, V., Tsuzuki, D. and Dan, I. (2007) 10/20, 10/10, and 10/5 Systems Revisited: Their Validity as Relative HeadSurface-Based Positioning Systems. NeuroImage, 34, 1600-1611. http://dx.doi.org/10.1016/j.neuroimage.2006.09.024

[39] Handy, T.C. (2005) Event-Related Potentials: A Methods Handbook. In: Handy, T.C., Ed., The MIT Press, Cambridge.

[40] Picton, T.W., Bentin, S., Berg, P., Donchin, E., Hillyard, S.A., Johnson, R. and Taylor, M.J. (2000) Guidelines for Using Human Event-Related Potentials to Study Cognition: Recording Standards and Publication Criteria. Psychophysiology, 37, 127-152. http://www.ncbi.nlm.nih.gov/pubmed/10731765 http://dx.doi.org/10.1111/1469-8986.3720127

[41] Green, C.S. and Bavelier, D. (2006) Effect of Action Video Games on the Spatial Distribution of Visuospatial Attention. Journal of Experimental Psychology, 32, 1465-1478. http://dx.doi.org/10.1037/0096-1523.32.6.1465

[42] Doehnert, M., Brandeis, D., Imhof, K., Drechsler, R. and Steinhausen, H.C. (2010) Mapping Attention-Deficit/ Hyperactivity Disorder from Childhood to Adolescence-No Neurophysiologic Evidence for a Developmental Lag of Attention but Some for Inhibition. Biological Psychiatry, 67, 608-616.

http://dx.doi.org/10.1016/j.biopsych.2009.07.038 